\title{
Unsichtbare Kindergeographien
}

\author{
Dominierende Denkfiguren von Kinderorten in der Stadt
}

\author{
Christian Reutlinger
}

Im Zuge der Covid-19-Pandemie wurden die unterschiedlichen Kinderorte in der Stadt neu geordnet. Kindergärten, Schulen, Freizeiteinrichtungen und Spielplätze wurden - zumindest temporär - geschlossen. Kinder waren auf ihren Wohnort zurückgeworfen. Bedeutsame Orte, die sie sonst selbstständig und in einer Gruppe von Gleichaltrigen erschlossen, waren ebenso außer Reichweite wie die Stadt als solche. Angesichts fehlender empirischer Studien zur Erklärung dieser veränderten Raumnutzung durch Kinder in der Stadt macht sich der Beitrag auf eine Spurensuche nach dominanten Thematisierungslinien bedeutsamer Kinderorte in der Tradition der sozialräumlichen Kinderforschung. Zeitlich und argumentativ macht die Suchbewegung drei dominierende Denkfiguren von Kinderorten in der Stadt sichtbar: Erstens die Großstadt ohne Kinderorte, zweitens die durchfunktionalisierte Stadt mit unpassenden Kinderorten und drittens die Stadt als Bildungskiste, in der sämtliche Kinderorte bildungsrelevant sind. Abschließend arbeitet der Beitrag die Gemeinsamkeiten dieser drei Denkfiguren heraus, überprüft sie auf ihren Erklärungsgehalt hinsichtlich der beschriebenen neuen Ordnung der Kinderorte in der Stadt und bestimmt übergangene Dimensionen.

Ersteinreichung: 30. November 2020; Veröffentlichung online: 26. November 2021

An English abstract can be found at the end of the document.

Im Frühjahr 2020 zeigte sich ein Phänomen, das mit den vorliegenden Deutungsangeboten zu Orten für Kinder in der Stadt nicht ohne Weiteres erklärbar ist. Der von vielen europäischen Regierungen ausgerufene sogenannte Lockdown in Reaktion auf die Covid-19-Pandemie führte zum Herunterfahren des öffentlichen Lebens sowie zur temporären Schließung von Kindergärten, Schulen, Freizeiteinrichtungen und Spielplätzen. Kinder waren in dieser Ausnahmesituation angehalten, teilweise wochenlang zu Hause zu verweilen, ohne eine Möglichkeit nach draußen zu gehen, Freund*innen zu treffen oder von Erwachsenen unbeaufsichtigt zu sein. Bedeutsame Orte, die sonst selbstständig und in einer Gruppe von Gleichaltrigen erschlossen und erlebten, waren für Kinder außer Reichweite. Die Stadt als solche war für sie mit einem Mal kaum mehr erfahrbar.

Zwar hat sich die Lage in den vergangenen Monaten in vielerlei Hinsicht entspannt, doch ist die Ordnung des Kinderlebens in der Stadt noch immer geprägt vom Krisenmodus. Bislang fehlen jedoch Studien und fundierte Daten, die Auskunft über die städtische Raumnutzung durch Kinder in der Krise geben. Unklar ist beispielsweise, wie sich Kinder durch den Lockdown 
ihren Wohnort neu angeeignet haben, welche Bildungsrelevanz dieser Ort erlangte und wie sich Schule als (un-)sicherer Ort hierzu neu in Beziehung setzte. Schließlich fehlt Wissen darüber, welche Konsequenzen hinsichtlich des Raumerlebens und der Raumerschließung diese (temporären) Einschränkungen etwa für unterschiedliche Altersgruppen oder in geschlechtsspezifischer Hinsicht haben. Zugleich scheinen neue Phänomene auf und werden mit raumindifferenten Deutungen erklärt. Dazu gehören etwa die in einigen europäischen Städten (darunter Brüssel, Stuttgart oder St. Gallen) plötzlich sichtbaren Formen der Raumaneignung durch Jugendliche oder jüngere Kids. Mit der Deutungsfolie Jugendgewalt werden diese Handlungen als Pöbelei, Zerstörungswut und Randalentum markiert. Die jungen Menschen werden in die Ecke sogenannter Corona-Leugner*innen oder Querdenker*innen gestellt. Dabei wird kaum hinterfragt, welche Bedeutung diese Prozesse der Aneignung öffentlichen Raums für die jungen Menschen haben und wie sie mit der veränderten Raumordnung in der Stadt zusammenhängen.

Eine empirische Forschung ist im Rahmen dieses Beitrags nicht möglich. Deshalb macht er sich auf die Spurensuche nach Deutungsmustern, mit denen die städtischen Raumnutzungen von Kindern, respektive Orte für Kinder in der Stadt bisher thematisiert wurden. Gelingt es damit, die beschriebenen aktuellen Phänomene zu verstehen? Setzt sich gar eine neue Deutung durch und eine damit verbundene gesellschaftliche und räumliche Ordnung von Orten für Kinder (insbesondere) in der Stadt? Und was bedeutet schließlich die (veränderte) Thematisierung für die Kinder und ihre Geographien im Sinne eigentätiger Welt-Bindungen, -Aneignungen und -Deutungen (Werlen 2015; Reutlinger 2017) in städtischen Kontexten?

\section{Denkfiguren: dominante Thematisierungen städtischer Kinderorte als Deutungsfolien von Kindergeographien}

Die Suche nach dominanten Thematisierungen städtischer Kinderorte erfolgt im Folgenden aus einer sozialräumlichen Kinderperspektive (Reutlinger 2014), die eine aktive Auseinandersetzung von Kindern mit ihrer sozialräumlichen Umwelt zum Gegenstand hat. Diese Perspektive beinhaltet stets zeitgleich zwei mögliche Akzentuierungen: erstens in Richtung einer „sozialräumliche[n] Konstitution von Kindheit als historisch-soziales Phänomen“ und zweitens in Richtung ,individueller Erfahrung“ sozialräumlicher Umwelterschließung durch Kinder (Honig 1996: 330). Auch wenn diese Unterscheidung in der aktuellen Forschung zu Kindern und Raum nicht immer so streng durchgehalten wird, mündet doch die erste Akzentuierung in eine sozialräumliche Kindheitsforschung (Andresen et al. 2016; BrachesChyrek/Röhner 2016; Schreiber 2014). Diese befasst „sich mit der Frage nach der Entwicklung und Veränderung der Lebensphase Kindheit und den damit zusammenhängenden Vorstellungen vom Kind in der Gesellschaft“ (Brüschweiler 2014: 44). Die zweite Akzentuierung, das Interesse nach der „subjektiven Einschätzung der einzelnen Kinder und auf ihre spezifische Wahrnehmung der eigenen Lebenswelt“ (Brüschweiler 2014: 44), führt hingegen zur sozialräumlichen Kinderforschung (Blinkert 1996; Deinet 1990; Kogler 2019). 
Die Suche bewegt sich zwischen diesen beiden Akzentuierungen, was zunächst eine Offenheit für verschiedene Zugänge zum Zusammenhang zwischen Kind, Raum und Umwelt zur Konsequenz hat. Das gilt in disziplinärer Hinsicht ebenso wie bezogen auf den jeweiligen Forschungsgegenstand im mittlerweile vielfältigen Diskursstrang zu Kinderräumen (Brüschweiler 2014: 26). Dieser Diskursstrang ist durch eine Vielzahl räumlicher - also je nach Raumverständnis physisch-materieller, symbolischer, gesellschaftlicher oder virtueller - Bedingungen des Aufwachsens geprägt, aber auch durch soziale, pädagogische, rechtliche, familiale oder entwicklungspsychologische Phänomene und disziplinäre Positionen (Reutlinger 2014). Im Zusammenwirken mit anderen Diskurssträngen stimmt der Diskursstrang zu Kinderräumen in einen Gesamtdiskurs zum Aufwachsen ein und konstituiert diesen mit. Angesichts dieser Komplexität bedarf es aus pragmatischen Gründen einer Einschränkung auf ganz bestimmte, typische Werke oder Texte mit einem sozialräumlichen Fokus. Es geht also um einen „Diskursausschnitt“ (Brüschweiler 2014: 52) beziehungsweise um eine Thematisierungslinie aus dem Diskursstrang Kinderräume, der oder die genauer analysiert wird, um zu „neuen Einsichten über räumliche Phänomene zu gelangen“ (Castillo Ulloa/Schwerer 2021: 415). Vor ähnlichen Herausforderungen standen das an der TU Berlin angesiedelte Forschungsprojekt „A02 Bildung: Raumwissen von Kindern und Jugendlichen in der Planung“ des DFG-Sonderforschungsbereichs 1265 „Re-Figuration von Räumen“ und die mit ihm verbundenen Überlegungen zur „qualitativen Meta-Analyse“ (Castillo Ulloa/ Schwerer 2021). Mit einer Meta-Analyse können „verschiedene, sonst eher getrennt betrachtete Forschungen“ und disziplinäre Perspektiven „zu einem gemeinsamen Phänomen“ zusammengefügt und daraus „neue Erkenntnisse“ generiert werden (ebd.: 425). Das im vorliegenden Beitrag angestrebte Ergebnis ist jedoch im Unterschied zu dem Berliner Projekt bescheidener. Das beginnt schon mit der Auswahl des Analysekorpus.[1] Dieser ist zwar, ebenso wie in dem Projekt, nicht disziplinär begrenzt, jedoch in sprachlicher Hinsicht. Er enthält ausschließlich deutschsprachige Texte (siehe Liste im Anhang).[2] Außerdem geht es nicht um die Generierung übergeordneten Wissens (wie etwa dem Raumwissen von Kindern und Jugendlichen, siehe Castillo Ulloa/Schwerer 2021), sondern um die Herausarbeitung von Konturen sogenannter „Denkfiguren“ (Reutlinger/Wigger 2010).

Eine Denkfigur dient erstens als „analytisches Instrument“ (ebd.: 15), zur Beschreibung der Verräumlichung von Kindheit (als sozialem und kulturellem Phänomen) und Kindern (sowie ihrem Erschließen konkreter Orte) in der Stadt. Zweitens dient eine Denkfigur als „Verständigungsfolie in [...] bisher unübersichtlichen sozialräumlichen Diskurs[en]“ zu Kindern und Raum (ebd.). Denkfiguren bleiben drittens nicht rein analytisch, sondern fließen über Praktiken und programmatische Konsequenzen zurück in den Diskursstrang. Als normativ fundierte Zuspitzung wird eine Denkfigur über unterschiedliche Diskursebenen (politisch, medial, programmatisch) in Praxen (re-)produziert und transformiert und wiederum diskursiv aufgenommen (Glasze/Mattissek 2009). Dies macht letztlich ihren konstitutiven Charakter für den Gesamtdiskurs aus - in diesem Fall jenem zum Aufwachsen.

Historisch betrachtet beginnt die sozialräumliche Auseinandersetzung mit Kindern in der Stadt am Übergang vom 19. zum 20. Jahrhundert. 1902 
rief die Reformpädagogin Ellen Key angesichts der zu dieser Zeit in der Industriegesellschaft in Bezug auf Kinder und deren Lebensräume herrschenden Missstände das Jahrhundert des Kindes aus (vgl. Key 2000 [1902]). Die Untersuchung nach der Art und Weise, in der Orte für Kinder in der Stadt thematisiert werden, beginnt hier und zieht sich bis in die Gegenwart, erstreckt sich also über einen Zeitraum von 120 Jahren. Die Spurensuche ist durch drei Momente charakterisiert, bei denen jeweils eine bestimmte Denkfigur die Thematisierung der Orte von Kindern in der Stadt dominiert, beziehungsweise daraus hervorgeht: Erstens der Moment der Entdeckung der Stadt oder genauer der „Großstadt“ als Lebensraum für Kinder zu Beginn des 20. Jahrhunderts (Abschnitt 1). Sie wurde zunächst ausschließlich als gefährlicher und gefährdender Kontext für Kinder thematisiert. Zweitens der Moment der Erschließung der Ortevon Kindern vor dem Hintergrund der hoch funktionalisierten Stadt der Nachkriegszeit (Abschnitt 2). Hier war Kindern und ihren Orten wenig Platz zugeschrieben. Dementsprechend mussten erst - meist überdachte, in der Regel pädagogisierte und dadurch kontrollierte - Orte eingerichtet werden. Und schließlich drittens der Moment der Eingemeindung der Orte von Kindern in die Stadt als Bildungsorte (Abschnitt 3). Im Sog der bildungspolitischen Auseinandersetzungen wurden die vielen Orte für Kinder unter bildungsrelevanten Gesichtspunkten diskutiert und die Stadt mit einer großen Bildungslandschaft gleichgesetzt. Im Zentrum der folgenden Analyse steht bei jedem der drei Momente ein Schlüsseltext, der den sozialräumlichen Diskurs weit über seine eigene Disziplin hinaus geprägt hat. Ergänzt wird dieser durch einige weitere Raumstudien, die zur Schärfung der jeweiligen Denkfigur beitragen können. Leitend sind dabei drei zentrale Anliegen einer sozialräumlichen Kinderperspektive: (1) Kinder werden als Expert*innen ihres Lebensraums betrachtet, den sie sich aktiv erschließen (Deinet 1990). (2) Das soziale Leben im Handeln und in der Organisation des Kinderlebens nimmt eine zentrale Bedeutung ein (Honig 1996: 332). (3) Trans- und interdisziplinäre Betrachtungsweisen sind von zentraler Bedeutung (Fritsche/Rahn/Reutlinger 2011). Die Einbeziehung zusätzlicher Texte, die in der jeweiligen Zeit rezipiert wurden, heute jedoch nicht mehr zum Kanon gehören, kann die jeweilige Denkfigur zusätzlich schärfen und bestimmte Aspekte stärker auf den Punkt bringen. In einem anderen Rahmen gälte es, diese Erweiterungen systematischer anzugehen, etwa indem insbesondere jugendkulturelle Aspekte oder sozio-ökonomische, geschlechtsspezifische sowie kulturelle Differenzlinien genauer herausgearbeitet werden. Zum Schluss jedes Abschnitts wird der Kern der Denkfigur noch einmal zusammengefasst.

Im Ergebnis werden drei Denkfiguren sichtbar, die zwar Diskursstränge und deren jeweilige Wirklichkeiten pointiert fassbar und darstellbar zu machen (Brüschweiler 2014: 45), jedoch - wie jede Systematisierung von Diskursen - modellhaft, situativ und damit flüchtig bleiben (Jäger 2012). Wie die folgende Analyse zeigt, erlangten diese Denkfiguren in einem bestimmten städtischen Kontext sowie auf Basis des Kindheitsdiskurses eine Stellung, „die sich zu einer bestimmten Zeit als dominierend durchgesetzt haben“ (Fritsche/Rahn/Reutlinger 2011: 17). Mit der Zeit wurden sie zwar von einer anderen Denkfigur überlagert, besitzen jedoch in aktuellen Diskussionen weiterhin Wirkmächtigkeit (Brüschweiler 2014), da sie ,gegenüber der Wirklichkeit eine Art Eigenleben führen. Sie stellen selbst Materialität 
sui generis dar" (Jäger 2012: 33). Die Vorstellung einer simplen Abfolge unterschiedlicher Denkfiguren greift daher zu kurz. Vielmehr kollidieren verschiedene Denkfiguren immer wieder miteinander.

Abgeschlossen wird die Spurensuche, die ihren Ausgangspunkt im beschriebenen Phänomen der Orte von Kindern unter den Bedingungen der Covid-19-Pandemie nahm, im 4. Abschnitt, der die drei Denkfiguren nochmals nebeneinander stellt, um aufzuzeigen, was immer schon übersehen beziehungsweise verdeckt wurde, nämlich die Kinder selbst und ihre Perspektive auf ihre Orte in der Stadt.

\section{Die Entdeckung der Orte von Kindern in der Stadt: Die Straße und der Lebensraum des Großstadtkindes}

Die Spurensuche nach der Frage, wie das Leben und Aufwachsen von Kindern in Städten thematisiert wird, beginnt in deutschsprachigen Großstädten am Übergang vom 19. zum 20. Jahrhundert. Antworten lassen sich anhand von zwei konkurrierenden Lesarten nachzeichnen:

Eine erste Lesart setzt an der funktionalistischen Betrachtungsweise des industriellen Großstadtlebens an. Lebensorte von Kindern werden dabei funktional-räumlich getrennt betrachtet. Sie beschränken sich auf „wohnen“ und „sich bilden“ (Maier et al. 1977: 129) als sogenannte „menschliche Daseinsäußerung“ (ebd.: 100). Solche Grundfunktionen menschlicher Daseinsäußerungen basieren auf Aktivitäten und Tätigkeiten, die „in allen sozialen Schichten immanent, massenstatistisch erfassbar, räumlich und zeitlich messbar sind und sich raumwirksam ausprägen“ (ebd.). Nach dieser bürgerlich geprägten Lesart finden Aufwachsen und Lernen exklusiv in der Familie und in der Schule statt. Als Institutionen, die die Kindheit prägten haben diese eine klar umrissene Funktion im gesamtgesellschaftlichen Gefüge sowie einen festen Platz im städtisch-funktionalen Siedlungszusammenhang. Diese Abtrennung besonderer Orte für Kinder im städtischen Siedlungszusammenhang kann als Zeichen einer „Herausbildung von Kindheit als einer besonderen gesellschaftlichen Lebensform “ interpretiert werden (Zeiher/ Zeiher 1994: 17): Junge Menschen wurden „in die privaten Funktionsräume der bürgerlichen Kleinfamilie und Schule verwiesen, um sie auf ihre spätere Aufgabe innerhalb der Gesellschaft vorzubereiten" (Schreiber 2014: 198).

Die zweite Lesart versucht, Orte von Kindern stärker aus dem Erleben des städtischen Alltags durch die Kinder selbst zu erschließen und dadurch Verbindungen zwischen den Daseinsbereichen herzustellen. Beide Lesarten fokussieren die Straße als sozialräumlichen Grenzbereich ganz unterschiedlicher Dynamiken und Einflüsse: einmal als Un-Ort des kindlichen Lebens und angesichts ihres Gefahrenpotenzials als pädagogisches Schreckgespenst für bürgerliche junge Menschen sowie einmal als vielschichtigen und wichtigen sozialen und räumlichen Zusammenhang des Aufwachsens und Lernens.

Die Straße als gesellschaftlicher Funktionsbereich der „Teilnahme am Verkehr“ und als Heimat des proletarischen Kindes

Funktionalistisch betrachtet nimmt Verkehr „eine Mittlerrolle zwischen den Standorten des Wohnens, des Arbeitsplatzes, der Versorgungs-, Bildungs- und 
Freizeiteinrichtungen, also zwischen den Funktionsstandorten" ein (Maier et al. 1977: 30). Mit dieser Funktion rückt vordergründig die Perspektive erwachsener, am Erwerbsleben beteiligter Stadtbewohner*innen in den Fokus. Der öffentliche Raum wird als exklusiver Bereich für Erwachsene betrachtet. Mit dem aufkommenden Einzug des motorisierten Nutzverkehrs in die industrielle Großstadt gehen vom Straßenraum zunehmend physische Gefahren für dort spielende beziehungsweise sich aufhaltende Kinder aus: überfahren, verletzt oder getötet zu werden ist für sie hier immer möglich. Zudem birgt die Straße als Aufenthalts- und Zufluchtsort ausgestoßener, mittelloser, deklassierter und nicht integrierter Menschen (Obdachlose, Prostituierte, Landstreicher*innen, Suchtmittelabhängige, Kriminelle etc.) nicht nur in physischer, sondern auch in sozialer Hinsicht ein Potenzial an Gefahren und Bedrohungen für Kinder.

In dieser Lesart nehmen Straßenjungen stellvertretend für andere Kinder die Rolle von „Rekruten des Bettelvolks, der Trinkerschar, des Verbrecherheers" ein (Rude 1915: 299). Diesen Aspekt machen insbesondere die Betrachtungen der Straße als Heimat des „proletarischen Kindes“ (Rühle 1911) im gleichnamigen Werk des (Sozial-)Pädagogen Otto Rühle deutlich (siehe Schlüsseltext $1 \mathrm{im}$ Verzeichnis des analysierten Datenkorpus). Rühle war einer der ersten zeitgenössischen Pädagog*innen, der das Straßenkindwesen sozialisationstheoretisch thematisierte. Er beschrieb die Folgen der Erlebnisse und Erfahrungen von Kindern auf der Straße sowie ihrer Erziehung durch die Straße für deren individuelle Entwicklung. Rühle rückte „die sozialen Missstände des großstädtischen Arbeitermilieus [...] und den unmittelbaren Einfluss der Großstadt auf die Psyche und das Bewältigungsverhalten proletarischer Kinder“ ins Zentrum (Stecklina 2002: $161 \mathrm{ff}$.):

„Das Großstadtkind [...] ist ganz auf die Straße angewiesen, die ihm Haus und Garten ersetzen muß. Es ist das Straßenkind - ein Produkt der Großstadt und der neuen Verhältnisse, ein neuer Typus. [...] Die Straße ist dem Straßenkinde in erster Linie Spielplatz. Im Gewoge und Getriebe zwischen Passanten [sic!] und Fuhrwerken aller Art, gestoßen und beiseite geschoben, angeschnauzt, von Schutzleuten verfolgt, von Gefahren bedroht, muß es sich beschäftigen und vergnügen, so gut es geht“"(Rühle 1915: 300).

Ebenfalls charakteristisch für die Beschreibung des Großstadtkindes ist die im nächsten Abschnitt ausgeführte Abgrenzung respektive das In-BezugSetzen zum Kind auf dem Dorfe, mit der auch Otto Rühle arbeitete.

\section{Die Straße als Anschauungsmaterial für schulisches Lernen und Lebensraum des Großstadtkindes}

Am Anfang der verstärkten Auseinandersetzung mit dem Leben und den Bedürfnissen von Kindern in der Großstadt steht die Gegenüberstellung des „Dorfkindes“ mit dem sich neu konstituierenden, noch wenig bekannten Typus des „Großstadtkindes“ (Gansberg 1907). Dies mündete in Stränge reformpädagogischer Gegendiskurse (Wandervogelbewegung, zurück zur Natur, zurück zur Familie) (Hopfner 2006). Die Umgebung, in der das Dorfkind aufwächst, wird als natürlich betrachtet, da ein „Leben in 
der Natur" gegeben ist (Gansberg 1907: 3). Auf dem Dorf können Kinder ursprüngliche, ganzheitliche und mit der Natur gekoppelte Erfahrungen machen. Die räumliche Umgebung der Großstadt wird hingegen als „unwirtlich“ (Mitscherlich 1965) und von der Natur, ja vom Menschsein entfremdet betrachtet. Eine solche Umgebung lässt Erfahrungen bloß unmittelbar und indirekt zu. Sie führt häufig auch zu aggressivem und destruktivem Verhalten junger Menschen (Mitscherlich 1965: 113). Mit der zunehmenden Funktionalisierung sind die Zusammenhänge ohne Gesamtverständnis voneinander getrennt, wie beispielsweise J. Müller in der Hamburgischen Schulzeitung festhält: „Ein Großstadtkind kennt in der Regel nur das fertige Produkt; ein Dorfkind kennt in der Regel auch die Entstehung des Produktes. Chemisch gesprochen: Die Vorstellungen eines Großstadtkindes sind größtenteils Verbindungen; die Vorstellungen des Dorfkindes in der Hauptsache Elemente." (Müller 1911: 36) Verschiedene Autor*innen leiten daraus - wie der zitierte Müller - vielfältige Herausforderungen für den schulischen Unterricht ab. Wo der Ansatzpunkt sein sollte, ist jedoch keineswegs klar. Für Müller stellen die „Uebelstände in der Hauptsache ein Stück Großstadtelend“ dar (ebd.). Daraus ergeben sich für ihn unterschiedliche Akzente des Unterrichts: Theoriebezug für Dorfkinder und Praxisbezug für Stadtkinder. Ganz anders die Sichtweise von Ernst Bläske, der in Reaktion auf Müllers Einwurf den Ansatzpunkt in den konkreten Lebensrealitäten und Erfahrungen von Großstadtkindern sieht - da diese den Kindern nicht fremd, sondern vertraut seien (Bläske 1911).

Nach und nach setzt sich die Einsicht durch, dass die städtischen Bedingungen, in denen Kinder aufwachsen, gar nicht defizitär, sondern vielmehr als großes Potenzial zu betrachten sind. Im Zuge dieser Entdeckung der Straße als anderem Lebenszusammenhang von Kindern entstehen beispielsweise die Streifzüge durch die Welt der Großstadtkinder (Gansberg 1907, siehe ergänzende sozialräumliche Studie - ESRS 1). Darin versucht der Volksschullehrer Fritz Gansberg in reformpädagogischer Absicht, die Schule zu öffnen beziehungsweise den schulischen Unterricht an die unterschiedlichen Lebensrealitäten und Erfahrungen von Kindern in der Großstadt anzupassen. Nach seinen Überlegungen kann ein „gesunder, anregender Unterricht“ nur im „alltäglichen Leben des Kindes“ wurzeln, „in der Kultur, die es umgibt“ (ebd.: 3). Stadtschulen sollten daher mit den „selbstgemachten Anschauungen des Kindes“ arbeiten und diese verarbeiten, also die Welt der Großstadtkinder zum Ausgangpunkt für den schulischen Unterricht machen.

Einen Schritt weiter geht einige Jahre später die Psychologin Martha Muchow in ihrer in Hamburg durchgeführten Studie Der Lebensraum des Großstadtkindes (Muchow/Muchow/Zinnecker 1998 [1935]), in der sie die kindliche „Lebenswirklichkeit Großstadt“ genau analysiert und beschreibt (ebd.: 71, siehe ESRS 2). Muchow untersucht mithilfe unterschiedlicher methodischer Zugänge sowohl den Raum „in dem das Großstadtkind lebt“, als auch den Raum „den das Kind erlebt“ (ebd.) sowie schließlich den Raum, „den das Kind lebt“ (ebd.). Die drei Perspektiven zusammen ergeben ein komplexes Bild von Orten in der Stadt, an denen sich Kinder aufhalten, von denen sie ihren Aktivitäten nachgehen und die sie durch Veränderung der „vorhandenen physisch-materiellen Arrangements“ zu ihrer Welt „umleben“ 
(ebd.: 123). Im Prozess des kindsspezifischen Umlebens der physischmateriellen Welt (wie etwa von Spielplätzen, verkehrsarmen Straßen oder Warenhäusern) entsteht eine personale Welt, die Muchow als Lebensraum bezeichnet. In der Entwicklung des Kindes dehnt sich der Handlungs- und Lebensraum langsam und kontinuierlich vom „mehr oder weniger eng um die Wohnung und die Wohnstraße“ verorteten Umfeld „schichtenförmig aus“ (ebd.: 147 f.). Die Struktur der kindlichen Lebensräume ist abhängig von „Geeignetheit als Spielgelände, Spielplatznähe, Bebauungsart, Nähe zum kindlichen Erleben usw.“ (ebd.: 148). Muchows Befunde hinterfragen die Idee, dass die ganze Großstadt als „Lebensraum des Großstadtkindes (als Raum, in dem das Kind lebt)“ (ebd.: 87) von Bedeutung sei. Vielmehr seien es nur bestimmte Orte und räumliche Ausschnitte. Der Lebensraum des Großstadtkindes baut sich nicht „neben dem der Großstadterwachsenen“ auf, sondern „überlagert“ oder „durchwächst“ diesen (ebd.).

Mitte der 1950er Jahre gelangt die Stadtsoziologin Elisabeth Pfeil in ihrer Studie Das Großstadtkind (Pfeil 1955, siehe ESRS 3) zu dem Schluss, dass eine „Charakterologie des Großstadtkindes“ fehlt (ebd.: 9). Sie setzt mit ihren Ausführungen an den Ergebnissen von Muchows Studie an, indem sie sich gegen die homogene Vorstellung eines „geschlossenen Typus“ Großstadtkind stellt und von einer sozialen und gebietsbezogenen Heterogenität der Großstadtbevölkerung ausgeht: „Innerhalb jeder Großstadt ist die Welt des Kindes eine ganz andere in jedem Stadtviertel und auf jeder Sozialstufe. [...] Und in der scheinbar uniformen Masse der Großstadtbevölkerung die Vielfalt und Fülle lebendiger Individualität, gerade bei den Kindern." (Ebd.: 11, s.o.)

\section{Zentrale Thematisierungslinien zum Zeitpunkt der Entdeckung}

In einer funktional gedachten und gebauten industriellen Großstadt wies man Kindern ganz bestimmte familiäre und schulische Orte zu. Außerhalb dieser war kein Platz für Kinder vorgesehen. Ihr Aufenthalt im Straßenverkehrsraum war zu gefährlich beziehungsweise zu gefährdend. Diese Sichtweise wurde in dem Moment revidiert, als die kindliche Welt der Stadt aus der Perspektive von Kindern gelesen wurde sowie als „konkrete[r] Raum [der Großstadt], in dem wir [beziehungsweise die Großstadtmenschen] ,drin“ sind“ (Muchow/ Muchow/Zinnecker 1998 [1935]: 147).

Durch das Umleben der physisch-dinglichen Welt konstituiert sich eine dem Subjekt zugeordnete Welt. Deshalb war für Muchow klar, dass es nicht darum gehen kann, zu untersuchen, ,wie eine so und so zu beschreibende Großstadtwelt die in ihr lebenden, so und so beschaffenen Kinder beeinflußte, sondern es war zu zeigen, wie das Kind seine Umgebung,Großstadt' zu seiner Umwelt umschafft und wie sich alsdann die vom Kinde, gelebte Welt' Großstadt darstellt“ (ebd.: 69). Der Lebensraum wurde jedoch nicht nur in seiner physisch-materiellen Dimensionierung betrachtet, nicht bloß als Ort der Kinder, sondern auch in seiner sozialen Räumlichkeit, als Ort, an dem sich Kinder ,auch Gesellschaft aneignen, sich mit Gesellschaft auseinandersetzten“ (ebd.: 174). Diese Verschränkung von Sozialem und Räumlichem war beispielsweise angelegt in der sich langsam ausdehnenden Welt, die sich das Großstadtkind erschloss und damit die ,räumlichen Konfigurationen der Gesellschaft" kennenlernte (Pfeil 1955: 12). 


\section{Erschließung (und Absicherung) der Orte der Kinder in der Stadt: Straßensozialisation und die kindliche Aneignung der Stadt}

Bei der Spurensuche nach der Auseinandersetzung mit Orten von Kindern in Städten lässt sich ab den 1970er Jahren eine Verschiebung feststellen: Das Aufwachsen auf dem Land sei besser, da ursprünglicher, natürlicher und unmittelbarer als in der Stadt (Zinnecker 2001), argumentieren Forscher*innen zum Lebens- und Aktionsraum von Stadtkindern. Das „Ineinandergreifen einer Reihe von Veränderungen“ (Zeiher 1990: 35) seit dem Zweiten Weltkrieg habe einen negativen Wandel der räumlichen Bedingungen des Aufwachsens zur Folge. Hervorgerufen werde dieser durch die Fokussierung aufTätigkeiten, die sich auf Erwerbsarbeit beziehen. Dazu gehörten insbesondere Konsumption und Verkehr. Diese prägten die Art und Weise der Siedlungsraumgestaltung. Das Auto wird dabei zum Herzstück der städtischen Entwicklung erklärt. Im Windschatten des motorisierten Verkehrs würden Kinder von der Straße vertrieben (Blinkert 2017). Wegen der fehlenden Anregungsmöglichkeiten verarmten Aneignungsprozesse. Ein spontaner Aufenthalt im Freien sei ebenso wenig möglich, wie unbeaufsichtigt draußen mit anderen Kindern zu spielen (Zeiher 1990: 37). Immer notwendiger werde die Schaffung von Reservaten und Ausgleichsangeboten wie beispielsweise Spielplätzen, Sportanlagen oder Kinderprogrammen (Blinkert 1996: 19).

Dieser zunehmende Bedeutungsverlust des öffentlichen Raums werde zusätzlich durch die Attraktivität von Binnenräumen gefördert, indem die organisierten Angebote zunähmen und ein immer ausdifferenzierteres, attraktives, den Alltag beherrschendes Medienangebot zur Verfügung stehe. Im Zuge dessen finde eine „Verhäuslichung von Kindern“ statt, jedoch auch eine räumliche und zeitliche Verinselung respektive eine institutionelle, räumliche und zeitliche Einschließung von Kindern (Zeiher 1990: 38). Angesichts dieser negativen Entwicklungen gelte es, die Bedeutungen der Orte von Kindern intensiv zu thematisieren, sie systematisch zu erschließen und dadurch abzusichern.

\section{Straßensozialisation, Kinderöffentlichkeiten und Selbstständigkeit}

Die Sicht auf Kinder und die Bedeutung ihrer Orte in der Stadt verändert sich grundlegend mit der Intensivierung der wissenschaftlich-programmatischen Auseinandersetzungen mit der Straße als „Lernort" sowie dessen Gleichsetzung mit den Lernorten Schule und Familie (Zinnecker 1979: 727). In seinem programmatischen Aufsatz Straßensozialisation. Versuch, einen unterschätzten Lernort zu thematisieren arbeitet der Erziehungswissenschaftler Jürgen Zinnecker Ende der 1970er Jahre ganz unterschiedliche Bedeutungsebenen dieses Lernortes heraus (siehe Schlüsseltext 2). Ausgangspunkt seiner Betrachtungen ist der konkrete oder „reale Ort“ Straße (ebd.), den gesellschaftlich betrachtet von Sechs- bis 18-Jährige besonders viel und intensiv nutzen. Betrachtet man jedoch nicht nur den „Verkehrsraum unter freiem Himmel“ (ebd.), sondern auch die „angrenzenden Räume und Gebäude“ (ebd.) - also verkehrsfreie Plätze, angrenzende Grünanlagen, Warenhäuser und Straßenkneipen -, so treten insbesondere der „öffentliche 
Raum" (Zinnecker 2001: 1) beziehungsweise unterschiedliche Konzeptionen von Öffentlichkeit hervor: Öffentliche Zugänglichkeit für jedermann ist dabei ein wesentliches Merkmal. Es unterscheidet diesen Ort von der Familie als „de[m] private[n] Ort“ (ebd.) und von Schule als halböffentlichem Ort.

Zinnecker nennt drei zentrale Bedeutungsgehalte des Lernortes Straße: Erstens wirke die Straße fördernd bei der Aufgabe „klassen-, geschlechts- und persönlichkeitsspezifische Identitäten und biografische Perspektiven aufzubauen“ (ebd.: 743). Zweitens diene der „gesellschaftliche Ort“ respektive die Auseinandersetzung mit anderen Menschen zum Erlernen von Kompetenzen für selbstständiges Auftreten und Handeln. Eine Straßenexistenz verlange schließlich drittens von jungen Menschen, „diesen funktionsbestimmten Ort für ihre Zwecke umzuwidmen, ihm eine neue Bedeutung zu verleihen, ihn auf Zeit zu ,kolonialisieren“" (ebd.: 741). Damit verweist Zinnecker auf die Wichtigkeit von Prozessen der Aneignung von Orten - sowohl für das Individuum als auch für Kindergruppen - und reiht sich damit in eine Vielzahl unterschiedlicher Studien ein, die ab Mitte der 1980er Jahre mit dem Begriff der sozialräumlichen Aneignung arbeiten und damit Orte von Kindern systematisch erschließen.

\section{Aneignung und die in den räumlichen Bedingungen der Orte liegenden Möglichkeiten}

Ausgangspunkt dieser sozialräumlichen Studien ist die Einsicht, dass konkrete Orte in der Stadt nicht nur von gegenständlich-räumlichen Bedingungen geprägt sind, sondern auch vom individuellen Handeln an diesen Orten. Der Begriff der Aneignung macht das „handelnde Subjekt“ (Jacob 1984: 687) zum Ausgangspunkt der Betrachtung. Er schließt „das Verändern, Umfunktionieren und Umdeuten der Umwelt“ (ebd.) in die Betrachtung ein. Exemplarisch dient der Begriff der Raumaneignung dazu, „das Verhältnis zwischen Kindern und Jugendlichen und der materiellen Umwelt in städtischen Räumen in städtischen Lebenswelten zu beschreiben“ (Harms/Preissing/Richtermeier 1985: 22).

Das Konzept der Aneignung betrachtet diekindliche undjugendlicheSozialisation als einen Prozess des Aufwachsens „in aktiver Auseinandersetzung mit der stofflich-dinglichen und sozialen Umwelt und mit sich selbst“ (Böhnisch 1996: 154). Durch ihn werde „die scheinbar tote sozialräumliche Welt der Gegenstände zu einer je individuellen sozialräumlich-personalen Anregungsstruktur" (ebd.). Lothar Böhnisch, aber auch Richard Münchmeier (2003), Ulrich Deinet (1990) oder Ursula Nissen (1998) betonen in ihren Arbeiten (siehe ESRS 4, 5, 6) immer wieder, dass es bei der Frage nach der Wirkung und Gestaltung der sozialräumlichen Umwelt nicht einfach um „Territorien oder Bauten, auf denen und in denen sich Menschen tummeln“ geht (Böhnisch 2002: 70). Vielmehr gehe es um „die Gelegenheitsstruktur, die Ressourcen, das Soziale, das in einem Sozialraum gegeben ist - das bestimmt die Lebensmöglichkeiten der Menschen“ (Münchmeier 2003: 3). Aus sozialpädagogischer Sicht werden deshalb räumliche Möglichkeiten im Sinne fördernder Umwelten gefordert, die Kinder und Jugendliche mit ihren Bedeutungen besetzen können: „Es ist nicht nur der Raum als territorialer Raum, der da sein muß, sondern die sozialen und emotionalen 
Möglichkeiten, die in ihm stecken und die von Kindern und Jugendlichen aus ihrer emotionalen Befindlichkeit und ihrem sozialen Wollen heraus erschlossen werden können.“ (Böhnisch 1996: 150)

Die Bedeutung der kindlichen Raumaneignung als tätiger Auseinandersetzung mit der räumlich-dinglichen Welt im Wohnumfeld unterstreicht auch der Soziologe Baldo Blinkert in seiner Untersuchung Aktionsräume von Kindern in der Stadt Anfang der 1990er Jahre (siehe ESRS 7). Im Fokus stehen Kinder zwischen fünf und neun Jahren, also einem Alter, in dem Kinder „anfangen, selbständig zu werden, [...] das Bedürfnis haben“, sich ihre „Umwelt auf eigene Faust zu erschließen, dabei Entdeckungen zu machen, unsere Neugier befriedigen und auch ungeheuer viel lernen“ (Blinkert 2017: 28). Wesentliche Hindernisse dabei beziehungsweise beim Erreichen von Spielmöglichkeiten im näheren Umfeld der Wohnung sind laut der Studie „Zugangsbeschränkungen und Gefahren durch den Straßenverkehr, von den Eltern befürchtete soziale Gefährdungen, sowie fehlende bzw. unzureichend gestaltbare Freiräume für Kinder“ (Blinkert 1996: 192). Blinkert fordert deshalb, das städtische Wohnumfeld solle eine „Aktionsraumqualität“ haben. Unter „Aktionsraum“ versteht er ein „Territorium außerhalb der Wohnung“, das über folgende vier Eigenschaften verfügt: 1. Zugänglichkeit für eine bestimmte Altersgruppe, das heißt „es darf keine sozialen, räumlichen oder verkehrstechnischen Barrieren geben“" (ebd.: 10). 2. müssen sich Kinder dort gefahrlos bewegen können - sowohl im objektiven wie im subjektiven Sinn (ebd.: 12). 3. Gestaltbarkeit: Kinder müssen also ihre „altersspezifischen Gestaltungsmöglichkeiten und -wünsche" in diesem Territorium umsetzen können (ebd.: 13). 4. Interaktionschancen: das Territorium „muß die Möglichkeit bieten, mit anderen Kindern zusammenzukommen“ (ebd.: 14).

\section{Zentrale Thematisierungslinien zum Zeitpunkt der Erschließung}

Als wichtiger Hinweis für die Stellung von Kindern in einer Gesellschaft lässt sich die wissenschaftlich-programmatische Auseinandersetzung mit der Straße als Aufenthaltsort von Kindern betrachten. Geht von der Straße bloß ein gefährliches beziehungsweise gefährdendes Potenzial aus oder wird die Straße als „Lernort“ und „Institution jugendlicher Erziehung und Sozialisation“ anerkannt (Zinnecker 1979: 735)? Die zweite Lesart thematisiert insbesondere das soziale Lernen. Dies beinhaltet beispielsweise den permanenten Austausch und die Konfrontation mit dem ambivalenten Charakter der Straße als Symbol für alles gesellschaftlich Unkontrollierte und nicht Integrierte. Darüber hinaus kann auf der Straße zentrale gesellschaftliche Rollen, wie derjenigen der öffentlichen Person oder die der Bürger*in eingeübt werden. Dazu gehört sowohl die Reibung mit Erwachsenen und der von ihnen vorgegebenen funktionalen Welt, also dem „bürgerlich-ökonomischen Funktionsraum“ (ebd.) als auch die Durchsetzung gegenüber anderen Kindern, also das Leben in Öffentlichkeit und Gemeinschaft, von Kinder- und Jugendkulturen. Daran schließen weiterführende Gedanken zum Unterschied zwischen der Öffentlichkeit Erwachsener und derjenigen von Kindern an, den sogenannten „Kinderöffentlichkeiten“ (Negt/Kluge 1972). Demnach bräuchten Kinder eine „raumbetontere Öffentlichkeit als Erwachsene“ (ebd.: 466). „Sie brauchen einen größeren Bewegungsspielraum, Plätze, die 
ein möglichst flexibles Aktionsfeld darstellen, in dem die Dinge nicht ein für allemal festgesetzt, definiert, mit Namen versehen, mit Verboten behängt sind.“ (Ebd.) Nur wenn nicht alles schon seinen unverrückbaren Platz, seine unumstößliche Ordnung habe, könnten Kinder selbsttätig werden, also Dinge zurechtrücken, Regeln und Normen umstoßen. Dies hebt einen zweiten wichtigen Aspekt der sozialräumlichen Aneignung hervor: Nicht die physisch-materielle Gestalt, die Dinglichkeit (der Objekte wie auch ihrer Anordnung) macht einen Ort aus, sondern die Möglichkeit, diese Funktionen umwidmen zu können, einem Ort neue Bedeutungen zu verleihen, ihn auf Zeit zu „kolonialisieren“ (ebd.).

Aus der Perspektive der Aneignung geraten zunächst nicht die (von Erwachsenen gestalteten und eingerichteten) Orte für Kinder in den Blick, sondern die Orte von Kindern beziehungsweise jene Orte, die für Kinder von Bedeutung sind: „Häufig sind sie nicht für Kinder gedachte oder geplante Orte, sondern vielmehr informelle und versteckte Räume: beispielsweise eine brachliegende Fläche zwischen Häuserblöcken oder Räume für Erwachsene, wie Einkaufszentren, die als gefährlich oder für Kinder ungeeignet gelten.“ (Kogler 2019: 12) Charakteristisch ist, dass diese Orte multifunktional sind oder sie ihrer vordefinierten Funktion gewissermaßen zweckentfremdet werden. Hierfür braucht es ein Anregungspotenzial oder die Möglichkeit einer individuellen oder gruppenspezifischen Aneignung (ebd.).

\section{Eingemeindung der Orte der Kinder in der Stadt: Viele Bildungsorte in der Stadt als Bildungslandschaften}

Ein dritter Moment bei der Spurensuche nach den Orten von Kindern in der Stadt ist der Beginn des 21. Jahrhunderts. Er wird hervorgerufen durch verstärkt neoliberal geprägte globale Wettbewerbs- und Verflechtungstendenzen. In der bundesdeutschen Bildungsdiskussion finden diese ihren Ausdruck in internationalen Vergleichsstudien (die sogenannten PISA-, TIMSS- und PIRLS-Studien[3]). Mit der Einsicht, dass schulische Bildung soziale Ungleichheiten nicht aufhebt, sondern verstärkt, geraten die Institution Schule als Ort von Integration und Selektion sowie das gesellschaftliche Versprechen einer gleichberechtigten Teilhabe aller Bevölkerungsgruppen in die Krise. Seither ist Bildung ein „Schlüsselthema“ (Rauschenbach 2005), sowohl in bildungspolitischen als auch in sozialräumlichen Auseinandersetzungen um Orte von Kindern in der Stadt. Ausgehend von der Schule und bezogen auf den schulischen Kompetenzerwerb werden sämtliche Orte von Kindern systematisch in den Blick genommen und auf ihre Leistungsfähigkeit hin überprüft. Zeitlich und örtlich werden diese Orte „vor, neben und nach der Schule" (Rauschenbach 2009: 210) in einen relationalen Zusammenhang gebracht: Vor der Schule tritt insbesondere die Familie als Bildungsort auf, da dort zumindest im Regelfall die Bildung der nachwachsenden Generation beginnt. Mit derselben Intention werden auch familienunterstützende und ergänzende Angebote wie die Kindertagesbetreuung unter Bildungsaspekten betrachtet. Neben der Schule wird insbesondere dem Kinder- und Jugendhilfesystem eine besonders wichtige Rolle als „Ort flexibler Bildung“ (Bock/Otto 2007) zugeschrieben. Schließlich werden die Bildungsräume und -strukturen nach der Schule, vom Jugendverein über das Kultur- und 
Sportangebot bis hin zum Spielplatz, erschlossen. Mit Letzteren geraten auch Orte von Kindern im öffentlichen Raum in den Sog der Bildung. Letztendlich steht die gesamte Stadt als förderlicher Zusammenhang für (informelle) Lern- und Bildungsprozesse im Fokus und wird unter dem Begriff oder im Namen der Bildung bewirtschaftet.

Neben der intensivierten Betrachtung der Orte von Kindern in der Stadt aus einer Bildungsperspektive kommt ein qualitativ neues Element dazu: die Betrachtung dieser Orte in der Zusammenschau. Diese vollzieht sich mit der Metapher der Bildungslandschaft (Reutlinger 2011), die nicht nur unterschiedliche Akteur*innen in den Blick nimmt, sondern darüber hinaus das Denken und die Verantwortungsübernahme für Bildungswege von Kindern in einer Gesamtschau anregt.

Kindsein in der Stadt aus der Exklusivität der BildungsbrilleBildungspolitische Programmatiken und fachliche Ergänzungen

Besonders bildungspolitische Programmatiken in der Bundesrepublik Deutschland propagieren die Gesamtschau formeller, nicht-formeller und informeller Bildungsorte als Gesamtzusammenhang. Eine Schlüsselstudie spielt hier der „Zwölfte Kinder- und Jugendbericht. Bericht über die Lebenssituation junger Menschen und die Leistungen der Kinder- und Jugendhilfe in Deutschland“ des Bundesministeriums für Familien, Senioren, Frauen und Jugend (BMFSFJ) von 2005 (siehe Schlüsseltext 3). Zwar definiert dieser Schule weiterhin als „wichtigste[n] Bildungsort moderner Gesellschaften“ (BMFSFJ 2005: 92), gleichzeitig unterstreicht er mantrahaft, dass „Bildung [...] mehr als Schule“ ist (ebd.: 12). Dementsprechend versucht der Bericht, die vielen und vielfältigen Bildungsorte und Lernwelten miteinzubeziehen. Neben Schule und Familie wird auch die Kinder- und Jugendhilfe als zentrale Akteurin beim „Ausbau eines flächendeckenden Systems für Bildung, Erziehung und Betreuung“(ebd.: 14) angesehen. „Die Kinder- und Jugendhilfe stellt mit ihren Leistungen und Angeboten vielfältige öffentliche Bildungsorte und -gelegenheiten bereit. Im Unterschied zur Schule handelt es sich hierbei jedoch durchgängig um freiwillig zu nutzende Angebote." (Ebd: 92) Schliesslich hebt der Bericht auch die zentrale Rolle von Medien sowie von „Gleichaltrigen-Gruppen, Peers und Cliquen“ für Bildungsprozesse hervor. Diese stellten „ein bedeutsames Erfahrungsfeld im Übergang von der Herkunftsfamilie in ein eigenständiges Netz sozialer Beziehungen“ dar (ebd.: 93).

Die hier skizzierten programmatischen Vorstöße werden unterstützt beziehungsweise fachlich unterfüttert durch eine verzahnte fachliche Diskussion beispielsweise von Seiten der Sozialpädagogik (Rauschenbach 2009). Diese warnt vor einer Verengung des Bildungsverständnisses im Mainstream-Diskurs, da „eine tradierte Orientierung auf Lernen, Wissen und Bildung im Sinne leistungsorientierter Komponenten“ dominiere (Otto/ Rauschenbach 2004: 5, siehe ESRS 8). „Was fehlt, ist ein Blick auf die andere Seite der Bildung, wie sie sich in den nicht-schulischen Bildungsorten und Bildungsmodalitäten im Kindes- und Jugendalter verdeutlicht.“ (Ebd.)

Dieser Anspruch einer ganzheitlichen Betrachtung führt letztlich dazu, dass die Stadt als Ganzes erneut in den Blick gerät, wie der Sammelband Stadtbaustein Bildung (Coelen/Heinrich/Million 2015) beispielhaft zeigt. 
Denn der städtische Raum hält „nicht nur bedeutsame Lernorte bereit“ (ebd.: 1, siehe ESRS 9). Vielmehr sei Bildung „von jeher eine der wichtigsten Funktionen von Städten“ (ebd.). Auf ähnliche Weise argumentiert auch der erwähnte Zwölfte Kinder- und Jugendbericht, indem er das Aufwachsen in Städten als Chance beschreibt, da sich in Städten „prinzipiell eine vergleichsweise hohe Vielfalt sozialer Erfahrungsmöglichkeiten durch unterschiedliche Betreuungs-, Freizeit- und Bildungsangebote sowie eine bunte Mischung unterschiedlicher Bevölkerungsgruppen und -schichten“ ergebe (BMFSFJ 2005: 70). Gleichzeitig beeinflussten die städtische Struktur sowie die sozio-ökonomische Lage der Familien „den Wohnstandort, die Wohnqualität und Wanderungsprozesse“, und damit die Frage, ob diese Chancen wahrgenommen werden können. Benachteiligend können sich laut des Berichts etwa sozialräumliche Bedingungen in sogenannten „sozialen Brennpunkten“ auswirken (ebd.): Hohe sozial-strukturelle Belastungsfaktoren (Arbeitslosigkeit, ethnische oder soziale Homogenität, auch in Schulklassen), gesellschaftliche Stigmatisierungsprozesse sowie das Fehlen positiver Vorbilder im sozialen Umfeld schränkten die „Möglichkeiten sozialen Lernens von Kindern und Jugendlichen in derartigen Quartieren“ ein (ebd.: 71).

\section{Zentrale Thematisierungslinien zum Zeitpunkt der Eingemeindung}

Die Schule als zentraler Bildungsort für Kinder ist Anfang des 21. Jahrhunderts in eine Krise geraten. Er muss im Innern reformiert werden und sich gleichzeitig nach außen öffnen. In breit angelegten bildungspolitischen Initiativen gilt es seither, sämtliche Orte von Kindern unter dem Fokus der Bildung neu in den Blick zu nehmen und in einen steuerbaren Gesamtzusammenhang zu bringen, die sogenannten Bildungslandschaften. Diefachpolitische Einführung der Begriffe Bildungsort und Bildungslandschaft kommt daher einer „(Wieder) Entdeckung der kommunalen Verantwortung von und für Bildung im lokalen Nahraum“ (Bollweg/Otto 2011: 20) gleich. Das Credo lautet: Stadt benötigt vielfältige Bildungsorte und -gelegenheiten, damit Kinder in ihren Bildungsprozessen gefördert werden - daher müsse auch der Blick auf die Orte von Kindern geöffnet werden. „Durch die vermehrte Berücksichtigung unterschiedlicher Bildungs-Settings gewinnen so auch Regionen und Städte, vor allem aber Stadtteile und Quartiere für die Bildungsdebatte an Bedeutung, da sie mit vielfältigen räumlichen Situationen und Gelegenheiten einen Rahmen für Bildungsprozesse setzen bzw. ihrerseits in solchen Prozessen gestaltet werden." (Million/Heinrich/Coelen 2015: 3) Mit dieser Geste geraten nun viele Orte von Kindern, die stets unter dem Radar der institutionalisierten Verwertungslogik liefen, vollständig in den Blick dominierender Bildungsbemühungen. In der Zusammenschau unterschiedlicher Bildungsorte und Akteur*innen geht es somit auch um eine Veränderung des Selbstverständnisses der einzelnen Bildungsorte. Mit Hinblick auf die Integration in ein Gesamtkonzept bedeutet das für sie, ,sich fortan als Teil des größeren und rahmengebenden Ganzen“ zu verstehen (Fritsche/Rahn/Reutlinger 2011: 56). Die Stadt wird damit zu einer riesigen „Bildungskiste“(Brüschweiler/Falkenreck 2019: 428), die es lediglich noch zu aktivieren beziehungsweise zu bestellen gilt. Aus dem Blick geraten dabei jedoch die Kinder und Jugendlichen selbst. Sie und ihre Geographien bleiben vielfach unsichtbar. 


\section{Abschließende Gedanken: Drei Denkfiguren von Kinderorten in der Stadt, ein verbindender Makel}

Die Spurensuche nach den Lesarten von Kindern und ihren Orten in der Stadt in den vergangenen 120 Jahren hat gezeigt, dass diese nicht einheitlich verliefen, sondern immer wieder auch konträr und vielfältig. Dennoch lassen sich drei dominierende Denkfiguren bestimmen, die sich zeitlich und argumentativ überlagern. Sie sind heute ein Deutungsangebot zur Beschreibung der städtischen Raumnutzung durch Kinder - mangels empirischer Daten auch unter den veränderten Bedingungen während der Covid-19-Pandemie:

Die erste Denkfigur ist die Großstadt ohne Kinderorte: Abgesehen von familiären und schulischen Orten ist nach dieser Lesart in der Großstadt kein Platz für Kinder vorgesehen. Diese Diagnose wird anhand der Straße als ambivalenter Sphäre schichtspezifisch und durchaus kontrovers diskutiert. Das „proletarische Kind“ (Rühle) wird auf der Straße sozialisiert und ist dort vielfältigen Gefährdungen ausgesetzt, während das bürgerliche Kind vor den Einflüssen der Straße beschützt wird. Studien zum Lebensraum des Großstadtkindes vermögen aufzuzeigen, dass sich Kinder nicht nur die physisch-materielle Welt aneignen, sondern im „Umleben“ (Muchow) auch die „räumliche Konfiguration der Gesellschaft“ (Pfeil) kennenlernen. Mit pädagogischen Motiven wird für die Schaffung von Lebenswelten plädiert, das heißt das Ideal naturnaher Orte für Kinder - orientiert am guten Leben auf dem Dorf - vertreten. Diese Orte werden in der Umsetzung jedoch monofunktional und rein physisch ausgestattet (beispielsweise durch das Aufstellen von Spielgeräten). Die Perspektive der Kinder und deren Eigentätigkeit bleiben damit außen vor - sowohl was die Anerkennung real von ihnen belebter großstädtischer Orte angeht als auch die für sie geschaffenen Orte. Mithilfe dieser Denkfigur ließen sich die aktuellen Veränderungen im Raumerleben dahingehend interpretieren, dass die Covid-19-Pandemie eine neuerliche Sensibilität erforderlich macht, um das „Umleben“ bisher eindeutig konnotierter Kinderorte unter den Bedingungen eingeschränkter Bewegungsfreiheit und Zugangsmöglichkeiten anzuerkennen und durch eine entsprechende sozialräumliche Forschung sichtbar zu machen.

Die zweite Denkfigur ist die der durchfunktionalisierten Stadt und der unpassenden Kinderorte: Ineinandergreifende negative Verkettungen der städtischen Siedlungsentwicklung führen demnach zu monofunktionalen, anregungsarmen Orten im öffentlichen Raum sowie zum Verschwinden von Freiflächen. Diese Prozesse führen zu einer Vertreibung von Kindern in verhäuslichte, institutionalisierte, verinselte, mediatisierte und oftmals von erwachsenen Bezugspersonen kontrollierte Orte. Studien zur Straßensozialisation und zur Aneignung durch Kinder zeigen die negativen Folgen dieser Entwicklungen auf und fordern mehr Möglichkeiten beziehungsweise Erlebnisqualität in der gebauten Welt, also ein Zulassen von Kinderöffentlichkeiten. Pädagogisch motiviert wird für die Schaffung funktionsoffener, aneigenbarer Orte plädiert, die jedoch in Form von Reservaten oder Ausgleichsorten als (Wald- oder Abenteuer-)Spielplätze, Sportanlagen oder Indoor-Kinderangebote umgesetzt werden. In der Tendenz entsteht dabei ein Netz aus Kinderorten in der Stadt, das sich an das Ideal der wohlgeordneten 
bürgerlichen Wohnung anlehnt. Die kindlichen lebensweltlichen Deutungen städtischer Orte werden wenig berücksichtigt. Sie bleiben trotz der intensiven Auseinandersetzung und der Schaffung der Orte von Kindern unberücksichtigt. Die zweite Denkfigur zeigt: Wenn zugängliche Orte wegfallen, wie während der aktuellen Covid-19-Krise, wird es umso wichtiger, nicht nur Anzahl und Ausstattung der Orte von Kindern in den Blick zu nehmen, sondern auch das Raumerleben und die Raumdeutungen durch Kinder konsequent zu verstehen und zu unterstützen.

Die dritte Denkfigur versteht Stadt als Bildungskiste, in der sämtliche Kinderorte bildungsrelevant sind: Bildung wird zum Leitthema, unter das sämtliche Kinderorte untergeordnet werden. Alternativ werden sie zur Schule als zentralem Bildungsort ins Verhältnis gesetzt. Mit dem Denken in Bildungslandschaften tritt die Thematisierung von Stadt als einem herausfordernden Lebenszusammenhang kindlichen Aufwachsens in den Hintergrund. Im Zuge dessen erhalten Kinderorte - egal ob eingerichtete oder gelebte - per se einen bildungsrelevanten Charakter und werden als „Stadtbaustein Bildung“ (Coelen/Heinrich/Million 2015) betrachtet. Empirische Studien greifen diese Denkfigur auf und stärken sie durch die die Betonung von Bindestrich-Pädagogiken, wie der subjektiven Bildung, der sozialen Bildung, der kulturell-musischen Bildung, der ästhetischen Bildung, der Medienbildung etc. Aus pädagogischer Perspektive werden nicht neue Bildungsorte oder bildungsfreie Orte gefordert, sondern eine Inblicknahme und Anerkennung der „anderen Bildungsorte“ (Otto/Rauschenbach) sowie der vielen Orte „vor, neben und nach der Schule“ (Rauschenbach). Kindliche Perspektiven und das kindliche Erleben von Orten in der Stadt geraten so in den Sog bildungsrelevanter Verwertungszusammenhänge und werden als eigentätige Leistung aberkannt. Die Art und Weise, wie Kinderorte in der Covid-19-Pandemie thematisiert wurden und werden, lässt jedoch vermuten, dass schulischer Bildung Systemrelevanz zugeschrieben wird, während allen anderen Gesellschaftsbereichen diese Relevanz abgesprochen wird. Kinder und ihre Praktiken der Ortsgestaltung - ihre Aneignung, Bedeutungsbelegung und ihr Eigensinn - geraten kaum in den Blick. Vielmehr drohen sie durch den ausschließlich bildungspolitischen Fokus auf den Ort Schule einmal mehr übersehen zu werden.

Die drei Denkfiguren verbindet, dass hinter ihnen jeweils eine bestimmte Art der Verräumlichung von Kinderorten und damit eine bestimmte gesellschaftliche Platzierung beziehungsweise Verortung von Kindheit steckt: Ohne das Zutun von Erwachsenen sind Orte in der Stadt für Kinder „schlecht“ beziehungsweise schädlich. Nur Erwachsene wissen, was „gute“ oder förderliche Orte sind. Sie können diese schaffen, indem sie sie von Schule und Familie aus denken. Auf diesem Wege kann eine „bessere“, kindergerechtere Stadt ermöglicht werden. Gemeinsam ist den drei Denkfiguren jedoch auch das Paradox, dass gerade durch die (intensivierte) Thematisierung der Kinderorte die Kinder selbst sowie deren Praktiken, sich mit der Welt zu verbinden und sie sich anzueignen - also die Orte aus Sicht der Kinder - an Bedeutung verlieren. Die intensivierte Auseinandersetzung mit Kinderorten in der Großstadt - in der durchfunktionalisierten Stadt oder in der Bildungsstadt - führt dazu, dass die Kindergeographien unsichtbar werden. Jeglicher Naturalisierung dieser Denkfiguren ist deshalb mit Kritik 
zu begegnen. Zugleich bietet die sich aktuell abzeichnende Neuordnung von Kinderorten in der Stadt eine Chance, die Kindergeographien jenseits dieser gängigen Deutungsfolien zu erforschen und damit der städtischen Raumnutzung und -gestaltung durch Kinder auf den Grund zu gehen.

Dieser Artikel wurde durch Mittel der Ostschweizer Fachhochschule (OST) gefördert.

\section{Endnoten}

[1] Die im Rahmen eines Expert*innenworkshops im Winter 2019 diskutierten Werke sowie die damit verbundenen Auswahlkriterien wurden für diesen Beitrag als Anregung für die Definition der Schlüsseltexte herangezogen, jedoch auf die Thematisierung von Orten für Kinder in der Stadt eingegrenzt.

[2] Diese sprachlich-diskursive Einschränkung reagiert auf einen noch immer bestehenden Mangel an sozialräumlichem Kinderdiskurs: Eine systematische Bezugnahme auf internationale Diskurse ist bisher noch die Ausnahme (Reutlinger/Brüschweiler 2016).

[3] PISA = Programme for International Student Assessment, TIMSS = Trends in International Mathematics and Science Study, PIRLS = Progress in International Reading Literacy Study.

\section{Autor_innen}

Christian Reutlinger ist Sozialgeograph und Erziehungswissenschaftler. Er forscht zu den Themen Bildung und Aufwachsen, Bildungsräume, Raumbilder.

chreutli@yahoo.com

\section{Analysierter Datenkorpus}

Denkfigur „Großstadt“

\section{Schlüsseltext 1:}

Rühle, Otto (1911): Das proletarische Kind. Eine Monographie. München: Albert Langen.

\section{Ergänzende sozialräumliche Studien (ESRS 1-3):}

Gansberg, Fritz (1907): Streifzüge durch die Welt der Großstadtkinder. Lebensbilder und Gedankengänge für den Anschauungsunterricht in Stadtschulen. Leipzig: Teubner.

Muchow, Martha / Muchow, Hans Heinrich / Zinnecker, Jürgen (1998 [1935]): Der Lebensraum des Großstadtkindes. Weinheim: Juventa Verlag.

Pfeil, Elisabeth (1955): Das Großstadtkind. Stuttgart: Ernst Klett.

\section{Denkfigur „Durchfunktionalisierte Stadt“}

\section{Schlüsseltext 2:}

Zinnecker, Jürgen (1979): Straßensozialisation. Versuch, einen unterschätzten Lernort zu thematisieren. In: Zeitschrift für Pädagogik 25, 727-746.

Ergänzende sozialräumliche Studien (ESRS 4-7):

Böhnisch, Lothar (1996): Pädagogische Soziologie: Eine Einführung. Weinheim: Juventa. 
Deinet, Ulrich (1990): Raumaneignung in der sozialwissenschaftlichen Theorie. In: Lothar Böhnisch / Richard Münchmeier (Hg.), Pädagogik des Jugendraums. Zur Begründung und Praxis einer sozialräumlichen Jugendpädagogik. Weinheim/München: Juventa, 57-70.

Nissen, Ursula (1998): Kindheit, Geschlecht und Raum: sozialisationstheoretische Zusammenhänge geschlechtsspezifischer Raumaneignung. Weinheim: Juventa.

Blinkert, Baldo (1996): Aktionsräume von Kindern in der Stadt: eine Untersuchung im Auftrag der Stadt Freiburg. Pfaffenweiler: Centaurus-Verlagsgesellschaft.

\section{Denkfigur „Stadt als Bildungskiste“}

\section{Schlüsseltext 3:}

Bundesministerium für Familie, Senioren, Frauen und Jugend [BMFSFJ] (2005): Zwölfter Kinder- und Jugendbericht. Bericht über die Lebenssituation junger Menschen und die Leistungen der Kinder- und Jugendhilfe in Deutschland. https://www.akzept.org/pdf/ volltexte_pdf/nr15/12.ki_jugendbericht.pdf (letzter Zugriff am 26.11.2020).

\section{Ergänzende sozialräumliche Studien (ESRS 8-9):}

Otto, Hans-Uwe / Rauschenbach, Thomas (Hg.) (2004): Die andere Seite der Bildung. Zum Verhältnis von formellen und informellen Bildungsprozessen. Wiesbaden: Vs Verlag für Sozialwissenschaften.

Coelen, Thomas / Heinrich, Anna Juliane / Million, Angela (Hg.) (2015): Stadtbaustein Bildung. Wiesbaden: Springer Fachmedien Wiesbaden GmbH.

\section{Literatur}

Andresen, Sabine / Fegter, Susann / Iranee, Nora / Bütow, Elena (2016): Doing urban family. Städtischer Raum und elterliche Perspektive auf Kindheit. In: Zeitschrift für Pädagogik 62/1, 34-47.

Bläske, J. F. E. (1911): Großstadtkind und Dorfkind. In: Hamburgische Schulzeitung 19/6, 43-44.

Blinkert, Baldo (2017): Kind sein in der Stadt. In: Sabine Fischer / Peter Rahn (Hg.), Kind sein in der Stadt. Bildung und ein gutes Leben. Opladen: Barbara Budrich, 27-48.

Bock, Karin / Otto, Hans-Uwe (2007): Die Kinder- und Jugendhilfe als Ort flexibler Bildung. In: Marius Harring / Carsten Rohlfs / Christian Palentien (Hg.), Perspektiven der Bildung. Kinder und Jugendliche in formellen, nicht-formellen und informellen Bildungsprozessen. Wiesbaden: Verlag für Sozialwissenschaften, 203-215.

Böhnisch, Lothar (2002): Räume, Zeiten, Beziehungen und der Ort der Jugendarbeit. In: deutsche jugend 50/2, 70-77.

Bollweg, Petra / Otto, Hans-Uwe (2011): Einleitung. Bildungslandschaft: Zur subjektorientierten Nutzung und topologischen Ausgestaltung. In: Petra Bollweg / Hans-Uwe Otto (Hg.), Räume flexibler Bildung. Bildungslandschaft in der Diskussion. Wiesbaden: VS Verlag für Sozialwissenschaften, 13-38.

Braches-Chyrek, Rita / Röhner, Charlotte (2016): Kindheit und Raum. In: Rita BrachesChyrek / Charlotte Röhner (Hg.), Kindheit und Raum. Opladen u. a.: Barbara Budrich, 7-33.

Brüschweiler, Bettina (2014): Die Rede von KinderRäumen. Eine kritische Diskursanalyse zu Kinder und Raum. Soziale Räume - Perspektiven, Prozesse, Praktiken, Band 2. https://www.fhsg.ch/fileadmin/Dateiliste/3_forschung_dienstleistung/institute/ifsar/ Publikationen/FHS_Publikation_Soziale_Raeume_Band_2.pdf (letzter Zugriff am 17.5.2021).

Brüschweiler, Bettina / Falkenreck, Mandy (2019): Bildungsorte und Bildungslandschaften. In: Fabian Kessl / Christian Reutlinger (Hg.), Handbuch Sozialraum. Sozialraumforschung und Sozialraumarbeit. Wiesbaden: Springer, 419-433.

Castillo Ulloa, Ignacio / Schwerer, Jona (2021): Qualitative Meta-Analyse. In: Anna Juliane Heinrich / Séverine Marguin / Angela Million / Jörg Stollmann (Hg.), Handbuch qualitative und visuelle Methoden der Raumforschung. Bielefeld: transcript, 415-425. 
Fritsche, Caroline / Rahn, Peter / Reutlinger, Christian (2011): Quartier macht Schule. Die Perspektive der Kinder. Wiesbaden: VS Verlag für Sozialwissenschaften.

Glasze, Georg / Mattissek, Annika (Hg.) (2009): Diskurs und Raum. Theorien und Methoden für die Humangeographie sowie die sozial- und kulturwissenschaftliche Raumforschung. Bielefeld: transcript.

Harms, Gerd / Preissing, Christa / Richtermeier, Adolf (1985): Kinder und Jugendliche in der Großstadt. Zur Lebenssituation 9-14-jähriger Kinder und Jugendlicher [Dissertation]. Berlin: TU Berlin.

Honig, Michael-Sebastian (1996): Probleme der Konstituierung einer erziehungswissenschaftlichen Kindheitsforschung. Ein Überblick über Fragestellungen, Konzepte und Befunde. In: Zeitschrift für Pädagogik 42/3, 325-345.

Hopfner, Johanna (2006): Aufwachsen in der Großstadt. Einige vergessene Positionen der Reformpädagogik. In: András Németh / György Mikonya (Hg.), Reformpädagogik und Lebensreform in Mitteleuropa. Budapest: Gondolat Kiadó Skiera, 204-220.

Jacob, Joachim (1984): Umweltaneignung von Stadtkindern. Wie nutzen Kinder den öffentlichen Raum? In: Zeitschrift für Pädagogik 30/5, 687-697.

Jäger, Siegfried (2012): Kritische Diskursanalyse. Eine Einführung. Münster: Unrast.

Key, Ellen (2000 [1902]): Das Jahrhundert des Kindes. Mit einem Nachwort von Ulrich Herrmann. Weinheim/München: Juventa.

Kogler, Raphaela (2019): Räume für Kinder - Räume der Kinder. Typologien urbaner Kinderräume. In: Bundesverband Wohnen und Stadtentwicklung, Forum Wohnen und Stadtentwicklung 1, 11-14.

Maier, Jörg / Paesler, Rainhard / Ruppert, Karl / Schaffer, Franz (1977): Sozialgeographie. Braunschweig: Westermann.

Million, Angela / Heinrich, Anna Juliane / Coelen, Thomas (2015): Schnittfelder zwischen Stadtentwicklung und Bildungswesen. In: Thomas Coelen / Anna Juliane Heinrich / Angela Million (Hg.), Stadtbaustein Bildung. Wiesbaden: Springer VS, 1-19.

Mitscherlich, Alexander (1965): Die Unwirtlichkeit unserer Städte: Anstiftung zum Unfrieden. Frankfurt am Main: Suhrkamp.

Müller, J. (1911): Großstadtkind und Dorfkind. In: Hamburgische Schulzeitung 19/5, 36-37.

Münchmeier, Richard (2003): Interview am 3. Dezember 2003 an der Fachhochschule Feldkirchen. http://cti.ac.at/cms/dateien/Interview_Muenchmeier.pdf (letzter Zugriff am 13.12.2009).

Negt, Oskar / Kluge, Alexander (1972): Öffentlichkeit und Erfahrung. Zur Organisationsanalyse von bürgerlicher und proletarischer Öffentlichkeit. Frankfurt am Main: Suhrkamp.

Rauschenbach, Thomas (2005): Plädoyer für ein neues Bildungsverständnis - Essay. https://www.bpb.de/apuz/29157/plaedoyer-fuer-ein-neues-bildungsverstaendnis-essay (letzter Zugriff am 24.11.2020).

Rauschenbach, Thomas (2009): Bildung - eine ambivalente Herausforderung für die Soziale Arbeit? In: Soziale Passagen 1/1, 209-225.

Reutlinger, Christian (2011): Bildungsorte, Bildungsräume und Bildungslandschaften im Spiegel von Ungleichheiten. Kritischer Blick auf das „Räumeln“ im Bildungsdiskurs. In: Petra Bollweg / Hans-Uwe Otto (Hg.), Räume flexibler Bildung. Bildungslandschaften in der Diskussion. Wiesbaden: VS Verlag für Sozialwissenschaften, 51-70.

Reutlinger, Christian (2014): Kinder und Raum aus sozialräumlicher Perspektive - ein Vorwort. In: Bettina Brüschweiler (Hg.): Die Rede von KinderRäumen. Eine kritische Diskursanalyse zu Kinder und Raum. Soziale Räume - Perspektiven, Prozesse, Praktiken, Band 2, I-VI. https://www.fhsg.ch/fileadmin/Dateiliste/3_forschung_dienstleistung/ institute/ifsar/Publikationen/FHS_Publikation_Soziale_Raeume_Band_2.pdf (letzter Zugriff am 17.5.2021).

Reutlinger, Christian (2017): Machen wir uns die Welt, wie sie uns gefällt? Ein sozialgeographisches Lesebuch. Zürich: Seismo.

Reutlinger, Christian / Brüschweiler, Bettina (2016): Sozialgeographien der Kinder. Eine Spurensuche in mehrdeutigem, offenem Gelände. In: Rita Braches-Chyrek / Charlotte Röhner (Hg.), Kindheit und Raum. Opladen u. a.: Barbara Budrich, 37-64.

Reutlinger, Christian / Wigger, Annegret (2010): Das St. Galler Modell. Eine Denkfigur zur Gestaltung des Sozialraums. In: Christian Reutlinger / Annegret Wigger (Hg.), Transdisziplinäre Sozialraumarbeit. Grundlegungen und Perspektiven des St. Galler Modelles zur Gestaltung des Sozialraums. Berlin: Frank und Time, 13-54. 
Rude, Adolf (1915): Schulpraxis. Unter besonderer Berücksichtigung neueren Bestrebungen und Einrichtungen. Osterwieck: Leipzig.

Schreiber, Verena (2014): Kindheit. In: Bernd Belina / Matthias Naumann / Anke Strüver (Hg.), Handbuch Kritische Stadtgeographie. Münster: Westfälisches Dampfboot, 195-200.

Stecklina, Gerd (2002): Otto Rühle und die Sozialpädagogik. Ein biografisch-sozialwissenschaftlicher Zugang [Dissertation]. Dresden: TU Dresden. https://d-nb. info/966504208/34 (letzter Zugriff am 24.11.2020).

Werlen, Benno (2015): Praktiken der Welt-Bindung. Gesellschaftliche Raumverhältnisse als trans-disziplinäres Forschungsfeld. In: Europa Regional 21.2013/1-2, 83-92.

Zeiher, Hartmut J. / Zeiher, Helga (1994): Orte und Zeiten der Kinder. Soziales Leben im Alltag von Großstadtkindern. Weinheim/München: Juventa.

Zeiher, Helga (1990): Organisation des Lebensraums bei Großstadtkindern. Einheitlichkeit oder Verinselung? In: Lothar Bertels / Ulfert Herlyn (Hg.), Lebenslauf und Raumerfahrung. Opladen: Leske und Budrich, 35-58.

Zinnecker, Jürgen (2001): Stadtkids. Kinderleben zwischen Straße und Schule. Weinheim: Juventa.

\section{Invisible children's geographies. Dominating figures of thought of children's places in the city}

In the course of the Covid 19 pandemic, various children's places in the city were reorganised. Kindergartens, schools, recreational facilities and playgrounds were closed - at least temporarily -, and children were confined to their homes. Significant places which children otherwise accessed independently or in peer groups, were out of reach, as was the city as such. In view of the lack of empirical studies which could explain this change in children's use of space in the city, the article sets out to trace dominant discussions of significant children's places in the tradition of socio-spatial children's research. Temporally and argumentatively, three dominant figures of thought regarding children's places in the city are traced: First, the big city without children's places; second, the thoroughly functionalised city with inappropriate children's places, and third, the city as an educational box in which all children's places are relevant to education. Finally, commonalities of these three figures of thought are worked out, examined for their explanatory power of the described new order of children's places in the city. Also, overlooked dimensions are determined. 